\title{
Evolution in circumstellar envelopes of Be stars: From disks to rings?^
}

\author{
Th. Rivinius ${ }^{1}$, D. Baade ${ }^{1}$, S. Štefl ${ }^{2}$, and M. Maintz ${ }^{3}$ \\ 1 European Southern Observatory, Karl-Schwarzschild-Str. 2, 85748 Garching bei München, Germany \\ 2 Astronomical Institute, Academy of Sciences, 25165 Ondřejov, Czech Republic \\ 3 Landessternwarte Königstuhl, 69117 Heidelberg, Germany
}

Received 25 June 2001 / Accepted 18 September 2001

\begin{abstract}
New series of echelle spectra were obtained to study the medium- and long-term evolution of the disks of several Be stars. Subtle variations in the wings of optically thin and thick emission lines suggest that the conventional, static picture of the disk being in quasi-contact with the central star is justified primarily (or perhaps only) after an outburst event. Some weeks to months later, a low-density region seems to develop above the star and slowly grows outwards. A subsequent outburst may later replenish this cavity. In fact, in two stars this more ring-like structure is apparently at times detached far enough from the star to allow for the formation of a secondary inner disk from the ejecta of a later outburst. This behaviour is not necessarily representative of Be stars in general because in the later spectral sub-types, discrete mass loss events have not so far been observed to play a major role. In the light of the apparent life cycle of such disks, a brief discussion is given of the differences in strength and variability between the winds of Be and normal B stars. It seems possible to attribute these differences to matter that was initially in the disk and therefore largely shielded from the stellar radiation, but that during the course of the inner excavation (or even complete distruction) of the disk becomes exposed.
\end{abstract}

Key words. stars: emission-line, Be - stars: circumstellar matter - stars: winds, outflows

\section{Introduction}

The circumstellar envelopes of Be stars still pose many challenges (Štefl 1999; Bjorkman 2000, for reviews). Some major improvements in understanding were accomplished only in the last few years. Most notably, the geometry of the envelope is now generally accepted to be a relatively thin disk (e.g. Hanuschik 1996; Quirrenbach et al. 1997, both deriving opening angles of less than $20^{\circ}$ ), and the velocity law in the disk was only a few years ago shown to be roughly Keplerian with very low non-orbital motions (e.g. Hanuschik 1996; Rivinius et al. 1999). On the other hand, the very origin of the disks is still a mystery. In some early-type Be stars discrete mass loss events may be triggered by the beating of nonradial pulsation modes (Rivinius et al. 1998b, for further discussion). But the fraction of the total mass content of a disk contributed

Send offprint requests to: Th. Rivinius,

e-mail: triviniu@eso.org

* Based on observations collected at the European Southern Observatory at La Silla, Chile, ESO proposal No. 64.H-0548 and on observations with the Wendelstein $80-\mathrm{cm}$ and the Ondřejov 2-m telescopes, both equipped with the HEROS spectrograph provided by the Landessternwarte Heidelberg. via such outbursts is unknown. Interestingly, rapidly rotating B stars without emission lines seem to be less prone to exhibiting detectable nonradial pulsation (e.g. Baade 2000).

Most models of Be star disks assume a relatively simple structure with the parameters varying monotonically with distance from the star. In particular, the radial density profile is typically approximated by a power law (e.g. Waters 1986; Millar \& Marlborough 1999)

Such a picture differs considerably from the ring-like models proposed in early papers explaining the origin and variability of line emission in Be stars. Struve (1931) suggests that the matter ejected at the equator due to critical rotation forms a nebulous ring, which revolves around the star and gives rise to emission lines. He mentioned Saturn's rings as a prototype of rings around Be stars. Struve demanded the envelope to be a ring as a consequence of his suggestion of supercritical rotation, rather than being based on observations. In Struve's picture, the gap would form naturally because of the angular momentum excess of the expelled material. He further pointed out that an elliptical ring might explain the Be stars with a variable violet-to-red peak intensity ratio (long term $V / R$ variables). 
Dynamical and physical properties of such rings were studied theoretically e.g. by Hazlehurst (1967), Limber \& Marlborough (1968) or Huang (1972). But the only observational motivation remained the hope to explain in this way the long-term $V / R$ variability. However, this was finally shown not to require a detached disk (see Okazaki 2000, for a recent review).

Although the variability of the line emission also was recognized almost right at the beginning of Be star research, most current models nevertheless omit all time dependencies. It is usually assumed that the associated restructuring of the disk can be described by some suitable sequence of stationary models. The only major exception is the inclusion of one-armed global oscillations responsible for the long term $V / R$ variability (Okazaki 2000, 2001, and references therein). However, they have so far been treated only as perturbations of an otherwise non variable, conventional disk. The increasing number of observations of rapid, discrete star-to-disk mass transfer events may now demand some refinement of the approach to variability.

These line emission outbursts seem to occur preferentially in, or are even confined to, Be stars of earlier spectral sub-types. One of the most detailed temporal profiles of such an event could be derived from observations of $\mu$ Cen. All outbursts observed in this star (Rivinius et al. 1998a, see also Sect. 2.1 below) basically follow the same generalized scheme:

Relative quiescence: for an extended period of time, the appearance of the emission lines does not change appreciably.

Precursor: within a few days, broad emission wings appear at the bottom of the HI line profiles, and the peak height of the emission lines drop slightly. Quasiperiodic variations in the ratio of the strength of the blue and the red emission peak ( $V / R$ variability) set in. The separation of the peaks in optically thin emission lines increases.

Outburst proper: the peak height not only recovers its former strength but reaches a temporary maximum and the $V / R$ variations terminate.

Relaxation: the extended wings disappear, and the peak height slowly decreases. Fe II and other metal emission lines may become undetectable and/or their peak separation starts to shrink again. This phase lasts much longer than either of the previous two. The rate of change of all parameters declines with time, and gradually a new phase of relative quiescence is entered.

In addition to this spectroscopic terminology, photometric variability is also observed connected to outbursts. For instance Hubert \& Floquet (1998) have shown that Be stars can undergo rapid brightness increases with subsequent slower fading on the same timescales as the abovedescribed outburst phenomena. Shell stars, being seen through the disk, in turn undergo brightness decreases with subsequent brightenings. This is explained in terms of the more massive disk after outburst contributing more continuum flux if seen pole-on (but of course also absorbing more if seen edge-on), and its dissipation.

Although partly much less complete, the observations presented in this paper of other stars are in very good agreement with this scheme. Therefore, the description given of them in Sect. 2.1 also uses the term "outburst". Additional justification of this notion is given in Sects. 2.2 and 3.

Rivinius et al. (1998a) revived the idea of a (temporary) ring, suggesting that in some cases the disk might at some times be detached from the star, instead of starting right at its photosphere, and be separated from it by a relative minimum in density. The disk would, then, look more nearly like a ring. This was derived from the varying width of disk emission lines of a single star, $\mu$ Cen. Meanwhile, more cases of such a behaviour were found (Rivinius 1999). This paper expands the corresponding database and, in addition, presents emission line profiles with features indicating the simultaneous presence of two separate structures, namely an inner disk and an outer ring.

From observations of the variable peak separation of the $\mathrm{H} \alpha$ line of $\lambda$ Eri, Smith et al. (1991) drew similar conclusions to those derived in this work. The same effect was also seen in $\mu$ Cen (Rivinius et al. 1998a).

More recently, Roche et al. (2000) also proposed a temporary ring structure from long-term variations of the He I emission lines of the X-ray binary X Per (cf. Sects. 2.1 and 3.3).

\section{Evolution of emission line profiles}

\subsection{Observations of individual stars}

The echelle spectra on which this work is based were secured during observing campaigns with the HERos (Štefl \& Rivinius 2000), and more recently also with the FEROS spectrograph (Kaufer et al. 1999). The main purpose of these observations was to investigate the short-periodic line profile variability $(l p v)$ of Be stars. Other publications are in preparation to discuss the observations in greater technical detail and in a broader context. This paper only focuses on the medium-and long-term development of emission lines between 400 and $850 \mathrm{~nm}$.

The current database comprises observations of the following stars (all spectral types given are from Slettebak 1982):

$\omega(28) \mathrm{CMa}(=$ HD $56139=\mathrm{HR} 2749$; B2.5 Ve): the temporal evolution of the maximum width of the emission lines is seen best in this star. It is one of the main targets of the $l p v$ program, having been observed from 1996 to 2000 (Štefl \& Rivinius 2000; Maintz et al. 2000; Stefl et al. 2000). Figure 1 shows the average spectrum from each observing run. An outburst in 1996 is well flagged by the broad H I emission wings and the observed variability (Štefl et al. 1998). Optically thin emission lines like Fe II 5169 are also quite broad. By the following year, the broad H I emission wings had disappeared and the disk emission lines had evolved to 

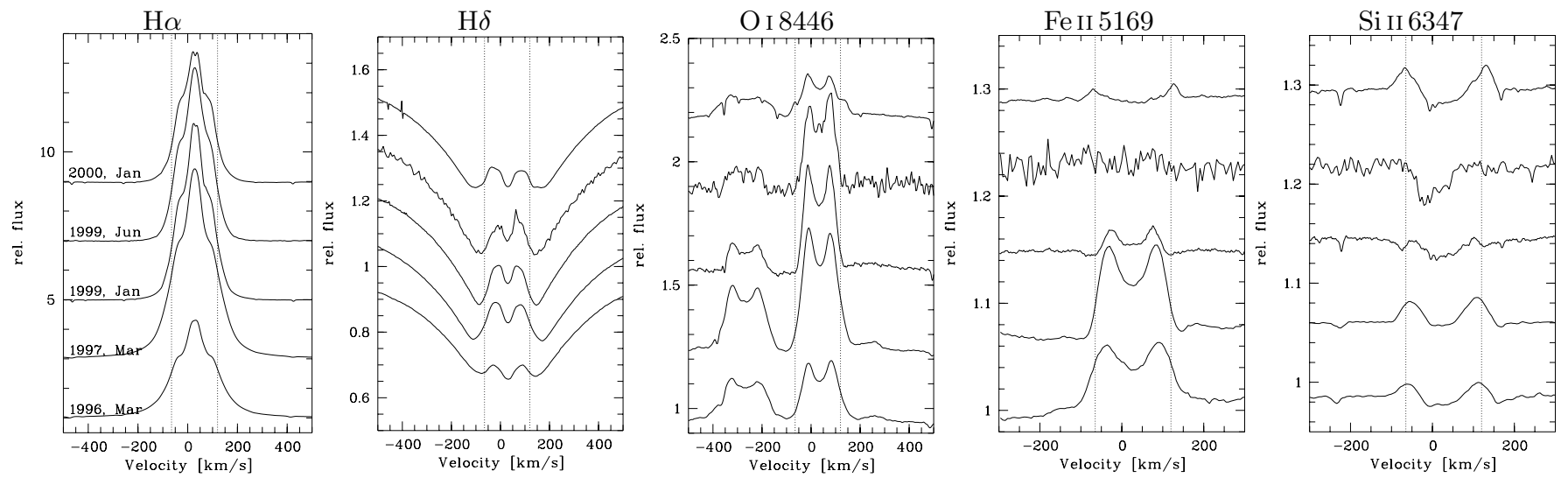

Fig. 1. The spectroscopic evolution of $\omega$ CMa from an outburst in 1996 to the next observed outburst in 2000. Averaged data are shown for each observing run. In 1999 June only a single spectrum was taken. Asymmetries in profiles from this observation are part of the short period stellar line-profile variability (Baade 1982); the averaging of the data has largely removed this from the spectra obtained in the other observing seasons. The panel for O I 8446 also covers Paschen 14. The dotted lines indicate the narrowest observed width of the optically thin emission lines at $[-65,+120] \mathrm{km} \mathrm{s}^{-1}$. Note that the scale of the velocity axes for Fe II and Si II differs from those of the other panels.

their maximum peak height. In January 1999, the hydrogen emission was still high, but the emission from the Fe II and Si II lines had significantly weakened and narrowed, and finally disappeared in the first half of 1999.

In the beginning of 2000, however, the Hi emission wings were present again, which is a reliable indicator of a new outburst à la $\mu$ Cen (Rivinius et al. 1998a). At the same time the Fe II and Si II line emission reappeared with a large width. The narrower doublepeaked emission in O I 8446 had lost a bit in width and two-thirds in peak height. But it had acquired a broad pedestal with the same total width as the other two lines. Like the $\mathrm{H} \alpha$ emission, this oxygen emission line is pumped by $\operatorname{Ly} \beta$ emission, but it is optically thin. It should, therefore, trace large parts of the disk without its profile being contaminated by self-absorption or scattering.

FV CMa (= HD $54309=$ HR 2690; B2 IVe): unfortunately, this star was observed during one observing run only so that nothing can be stated about a possible temporal evolution. Thirteen spectra were taken with FEROS at the ESO $1.52-\mathrm{m}$ telescope over a period of 17 days in January, 2000. All spectral features discussed here were stable during the entire observing period. Therefore, the data were averaged. The emission in the Balmer lines shows two quite clearly separated constituents. In $\mathrm{H} \beta$ a strong narrow and a broad weaker component are apparent. Towards the higher Balmer lines, the narrow component weakens more rapidly than the broad one. At their base, the total widths of these two constituents are $415 \mathrm{~km} \mathrm{~s}^{-1}$ and $670 \mathrm{~km} \mathrm{~s}^{-1}$, respectively (Fig. 2).

FW CMa (= HD $58343=$ HR 2825; B3 Ve): the variable base width of the emission in Fe II is clearly visible in observations extending over the years 1996-2000 (Fig. 3). Also the emission peak seperation is variable in the same sense as in $\omega$ CMa (Fig. 1) The timescales are, however, long. For instance, there was only a slight decrease in peak height from 1996 to 1997, but no width variation at all. In 1999, the Fe II 5169 line had turned into absorption, with some central emission component still seen, however. In 2000, when Fe II 5169 was in emission again, the $\mathrm{H}$ I lines had also developed broad emission wings.

$\mu$ Cen (= HD $120324=$ HR 5193; B2 IV-Ve): Figure 4 complements Figs. 1, 5, 6, and Sect. 7 in Rivinius et al. (1998a) and extends them in temporal coverage. Especially in Fig. 5 of Rivinius et al. (1998a, panel for Si II) it can well be seen how the narrower emission line of an older circumstellar structure is complemented during an outburst by a broader emission component. By 1999, only the Balmer lines have retained their emission at their approximate previous strength. Even the Paschen and O I emissions have nearly vanished, and all other emission lines are no longer detectable.

$\eta$ Cen (= HD $127972=$ HR 5440; B2 IV (e)): the shell nature of this star makes it difficult to determine the position of emission peaks in weak lines. But an obvious sudden change occurred in February, 1996 (Fig. 5), when the maximum base width of the He I 6678 emission increased from $[-330,+350] \mathrm{km} \mathrm{s}^{-1}$ to $[-540,+560] \mathrm{km} \mathrm{s}^{-1}$. A similar increase was seen in the Balmer lines, although numbers are hard to give because of electron scattering blurring the footpoints of the emission.

$o$ And (= HD $217676=$ HR 8762; B6 III): among the Be stars, in which outbursts have been seen, o And has (one of) the latest spectral subtypes. The $\mathrm{H} \alpha$ emission developed a second component at higher velocities from the end of August, 2000 to mid September (Fig. 6; the star was not observed in the beginning of September). From September on, transient period-type 

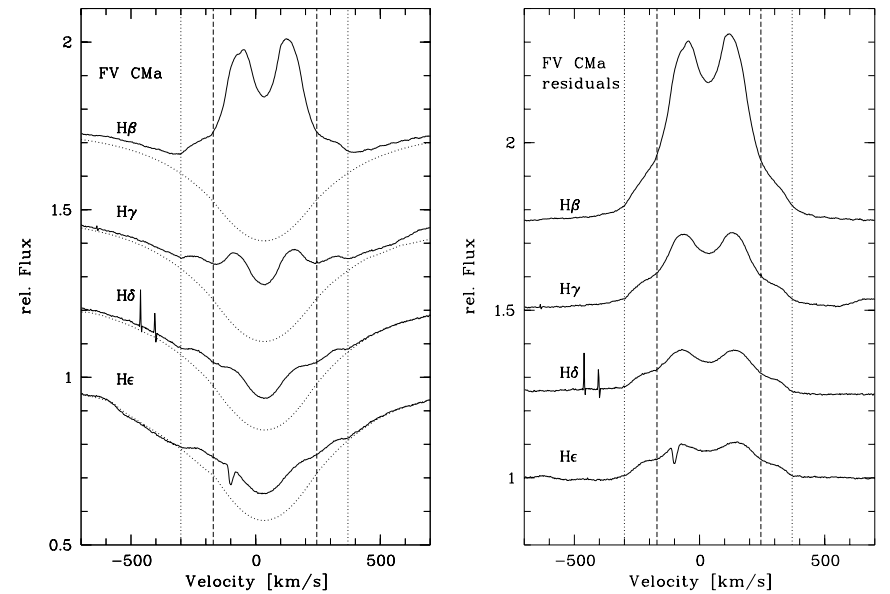

Fig. 2. The spectroscopic evidence for two spatially separate disk constituents in the average of 13 spectra of FV CMa obtained in 2000 January. The residuals were computed by subtracting a synthetical spectrum with typical B2 IV parameters $\left(T_{\text {eff }}=21000 \mathrm{~K}\right.$ and $\left.\log g=3.7\right)$, broadened to $v_{\text {rot }} \sin i=$ $200 \mathrm{~km} \mathrm{~s}^{-1}$. The observed spectra were corrected for the star's systemic velocity of $v_{\text {sys }}=35 \mathrm{~km} \mathrm{~s}^{-1}$. Starting from $\mathrm{H} \gamma$ a second, wider pair of emission peaks appears to emerge towards the higher Balmer lines. The edge velocities of each constituent were estimated to $[-300,+370] \mathrm{km} \mathrm{s}^{-1}$ for the wide and to $[-170,+245] \mathrm{km} \mathrm{s}^{-1}$ for the narrow one and are indicated by vertical lines. The Balmer decrement is significantly shallower for the broad constituent. Residual CCD artifacts are seen on the blue side of $\mathrm{H} \delta$, as well as the interstellar Ca II line in $\mathrm{H} \epsilon$.

variability (Štefl et al. 1998, rapid $V / R$ variations) was seen, although the data are not sampled well enough to derive a period, especially during the beginning of the outburst.

The metallic shell absorption observed in 1998 (Rivinius et al. 1999) has almost completely disappeared. Similarly, the Paschen shell absorption cores have weakened, but the Balmer absorption profiles are largely unchanged between 1998 and 2000. This behaviour is typical of the evolution of Be star disks, see e.g. $\mu$ Cen and $\omega$ CMa.

28 Cyg (= HD $191610=$ HR 7708; B3 IVe): a very similar behaviour of varying Fe II emission line width and multiple outbursts as in $\mu$ Cen was reported by Tubbesing et al. (2000, their Fig. 1), based on 1997 and 1998 data. Meanwhile, new observations in spring/summer 2000 (with Heros at the Wendelstein 80-cm telescope) and fall 2000 (with HERos and the Ondřejov 2-m telescope) have confirmed this behaviour. Interestingly, the high velocity tails have disappeared, but the position of the emission peaks remained largely unchanged in Fe II 5169 (Fig. 7).

A search of the literature showed that DU Eri $(=228$ Eri $=$ HD $28497=$ HR 1423; B1 Ve) was once reported to display an unusual $\mathrm{H} \alpha$ profile (Hanuschik et al. 1996, their Fig. 10d). In comparison to other observations of the same star, the inner two emission peaks were relatively narrow and arose above a weaker but much broader emission

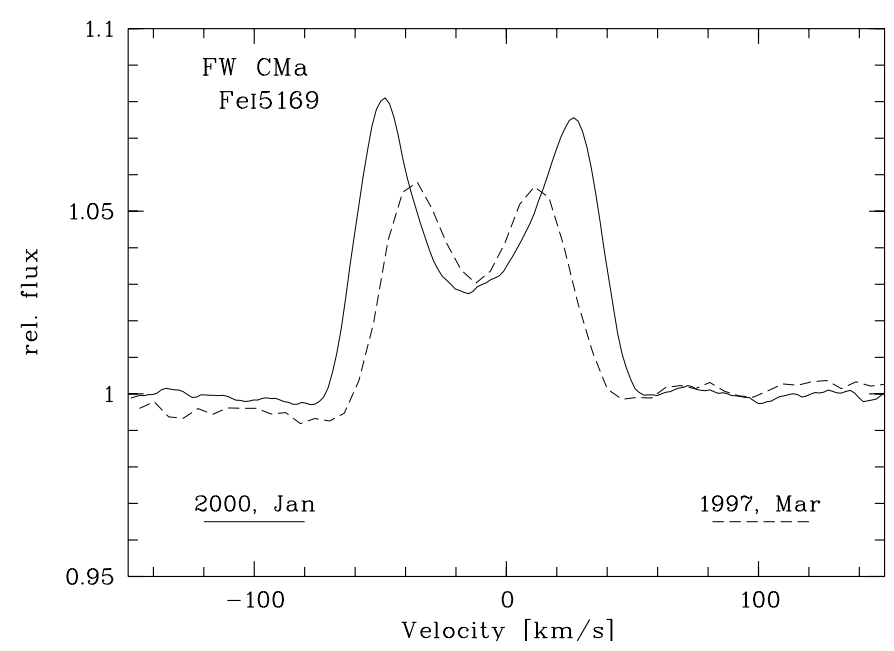

Fig. 3. FW CMa behaves very similarly to $\omega$ CMa (Fig. 1), except that no broad base in the O I 8446 emission line is seen. Only spectra exhibiting the extreme maximum and minimum width, respectively, of the Fe II 5169 emission observed between 1996 and 2000 are shown.
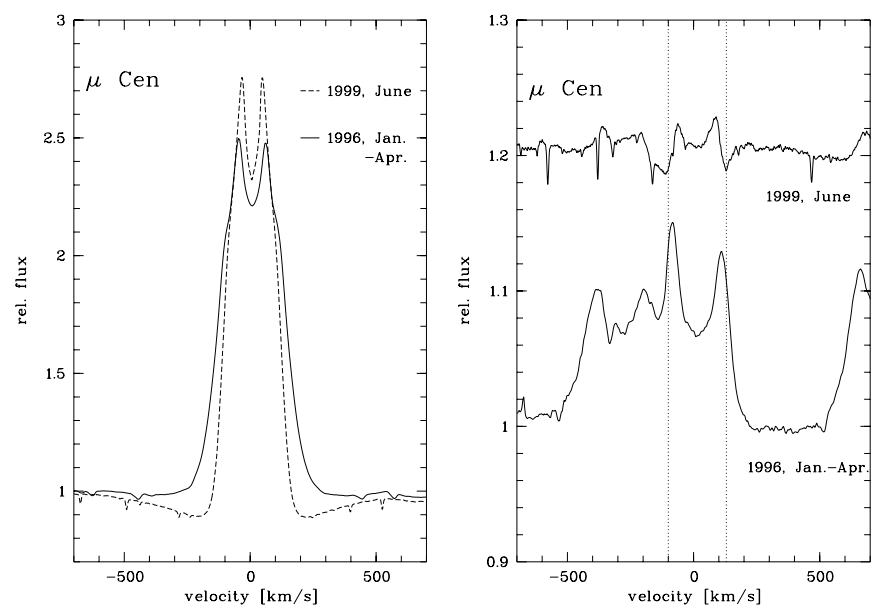

Fig. 4. The $\mathrm{H} \alpha$ and Or 8446 emission lines of $\mu$ Cen in 1996 and 1999. The narrowest width of OI8446, $[-100,+130] \mathrm{km} \mathrm{s}^{-1}$, is indicated by vertical lines. The variability of the broad $\mathrm{H} \alpha$ wings is similar to that in $\omega \mathrm{CMa}$ (Fig. 1).

constituent highly akin to the dual structures in $\omega \mathrm{CMa}$ and FV CMa. Feros spectra taken of DU Eri in January 2000 also show some structure in $\mathrm{H} \beta$ similar to the same line in FV CMa (Fig. 8).

Waters \& Marlborough (1994, their Fig. 1) reported a broad emission plateau in the $\mathrm{Br} \gamma$ line of $\psi$ Per, observed in 1992, to which the normal Be double peak profile was superimposed. They described the plateau as symmetric and disappearing in Br $\alpha$. Electron scattering as the process of formation is ruled out because of the sharp edges separating the plateau and normal emission profile. From this, a formation of the plateau in the part of the disk close to the stellar surface is suggested.

Observations by Smith et al. (1991) of variable peak separation in the $\mathrm{H} \alpha$ profile of $\lambda$ Eri also fit the above picture well, and were interpreted already by these authors as a ring. However, this could also be understood in terms of a variable outer disk radius and without further support 

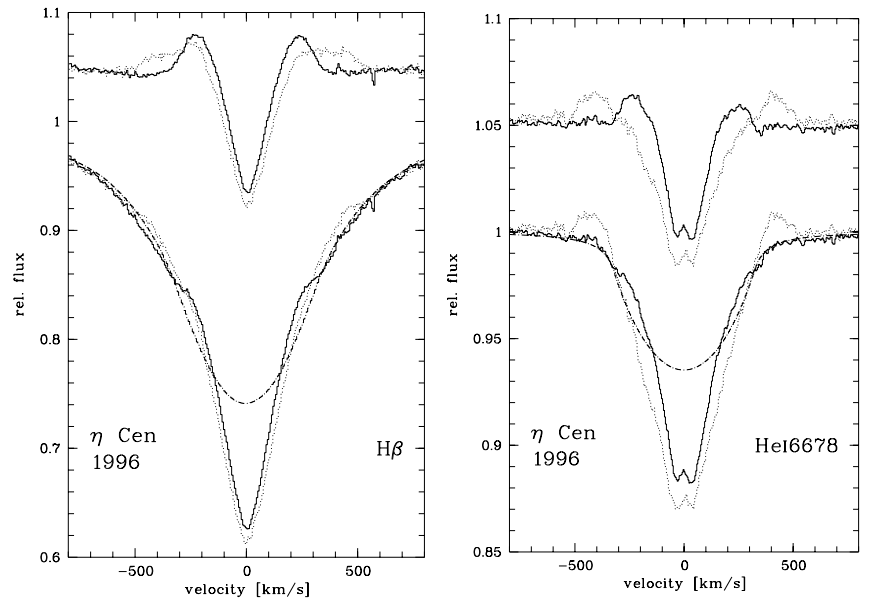

Fig. 5. The $\mathrm{H} \beta$ and He 6678 lines of $\eta$ Cen in 1996 before and after an outburst. To improve the $S / N$ ratio, the averages of 19 days before the burst (solid, MJD=50101 to 50119 ) and the 5 days after (dotted, 50121 to 50126 ) are shown. No spectrum was taken on 50120 . A synthetic spectrum, using the parameters by Balona (1999, dash-dotted profile, $25000 \mathrm{~K}$, $\left.\log g=4.0, v \sin i=331 \mathrm{~km} \mathrm{~s}^{-1}\right)$ was subtracted to make the differences of the emission more visible (residuals plotted above the spectra). The central quasi emission (CQE) in He I 6678 is a purely kinematical effect of the rotating disk seen edge-on (Rivinius et al. 1999) and indicative of the outer radius of the disk. The invariance of the CQE, the visibility of which depends strongly on stable (non-turbulent) conditions, shows well that the ouburst is confined to the innermost parts of the disk.

is not sufficient to unambiguously establish a detached ring.

$\mathrm{X}$ Per (= HR $1209=$ HD 24534) is a highly active X-ray binary with a probable orbital period of $250 \mathrm{~d}$ (DelgadoMartí et al. 2001) and consists of a B0e primary and a neutron star. Observations by Roche et al. (2000) and spanning a decade of the variability of the emission in $\mathrm{H} \alpha$ and He I 6678 are dominated by the initial complete loss of the disk, its later recovery, and large-scale $V / R$ variations. After abstraction from these phenomena, the relatively quick apperance and slower decay of broad emission wings closely resembles the outbursts described above for other, single Be stars. Still more interestingly, at several epochs the He 6678 emission displayed a broad plateau underlying a narrower double-peaked emission. In at least one instance, this plateau even rose to two discrete outer peaks, which were yet more pronounced than in $28 \mathrm{CMa}$, FV CMa or DU Eri.

\subsection{Synthesis of a common pattern}

Any attempt to understand the above bewildering diversity of details must start with a preliminary taxonomic effort to identify commonalities, although future observations will undoubtedly modify the summary developed in the following. In any event, its scope is presently limited to this particular sample of Be stars.

The primary feature is the variability of the base width of emission lines. This has been known for a long time.

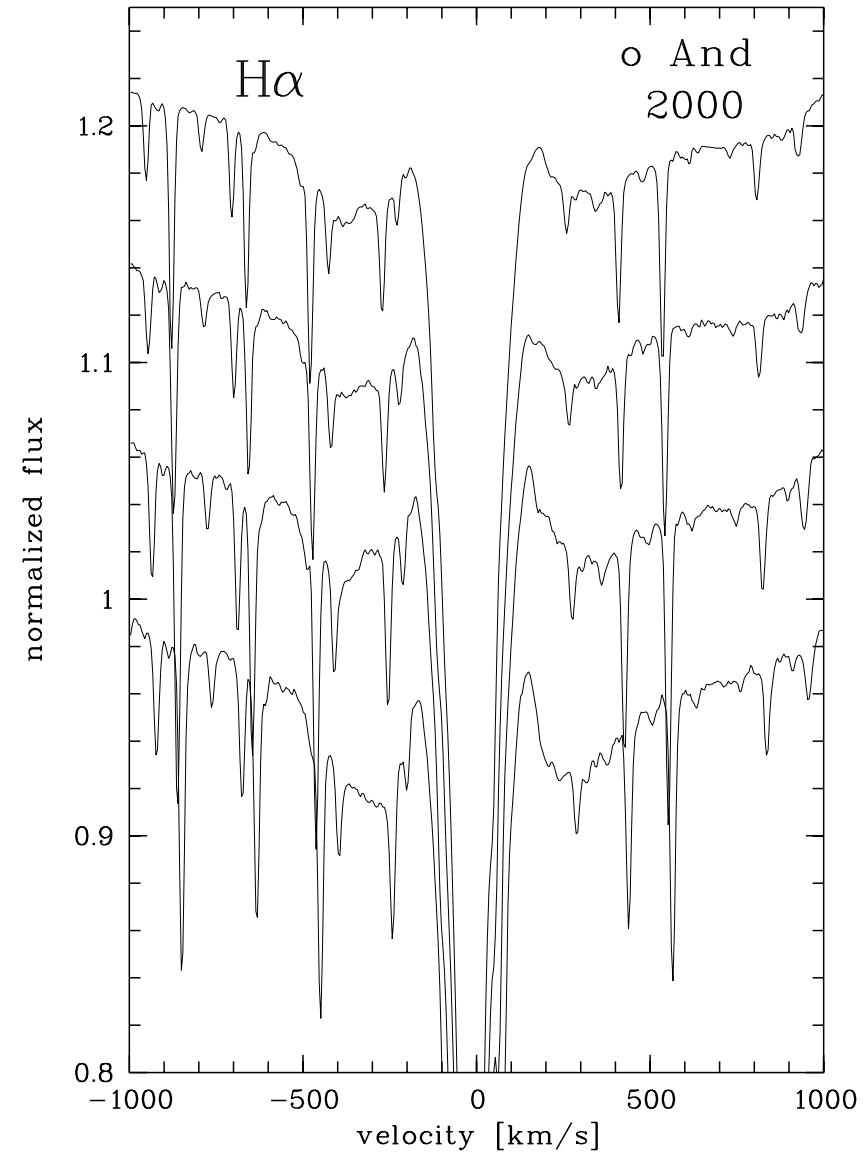

Fig. 6. $o$ And showed signs of a typical outburst in the second half of 2000. The figure demonstrates the increase of $\mathrm{H} \alpha$ emission wings from 74 spectra taken from August to November 2000. The spectra have been averaged in roughly 20-day intervals (from bottom to top: MJD 51 766-783, 51 798-816, 51 826845, and 51 855-875). While the star was quiet in August (until MJD 51783 ), clear additional emission appeared at higher velocities around mid September (there were no observations in the beginning of that month). The $V / R$ ratio of this emission was highly variable on timescales of days or shorter for some time. Unfortunately, not enough spectra were taken in this phase that a time series analysis could be attempted. When the star was observed more frequently in November the rapid $V / R$ variability had already settled. However, this rapid $V / R$ variability excludes an interpretation by electron scattering, since this should produce a symmetric profile. The numerous sharp absorptions are due to telluric water vapour. Their radial velocity variation reflects the changing heliocentric correction during the observing campaign

So, the main interest lies in the following refinements of this information:

- there is no restriction to optically thick lines. In a given Be star, all emission lines are affected, albeit with much gradation: Weak, optically thin lines may entirely vanish or (re-)appear whereas in optically thick lines the variation relative to a strong mean emission may easily not be recognized;

- such variations can occur in perfectly symmetric profiles and independently of long-term $V / R$ cycles; 


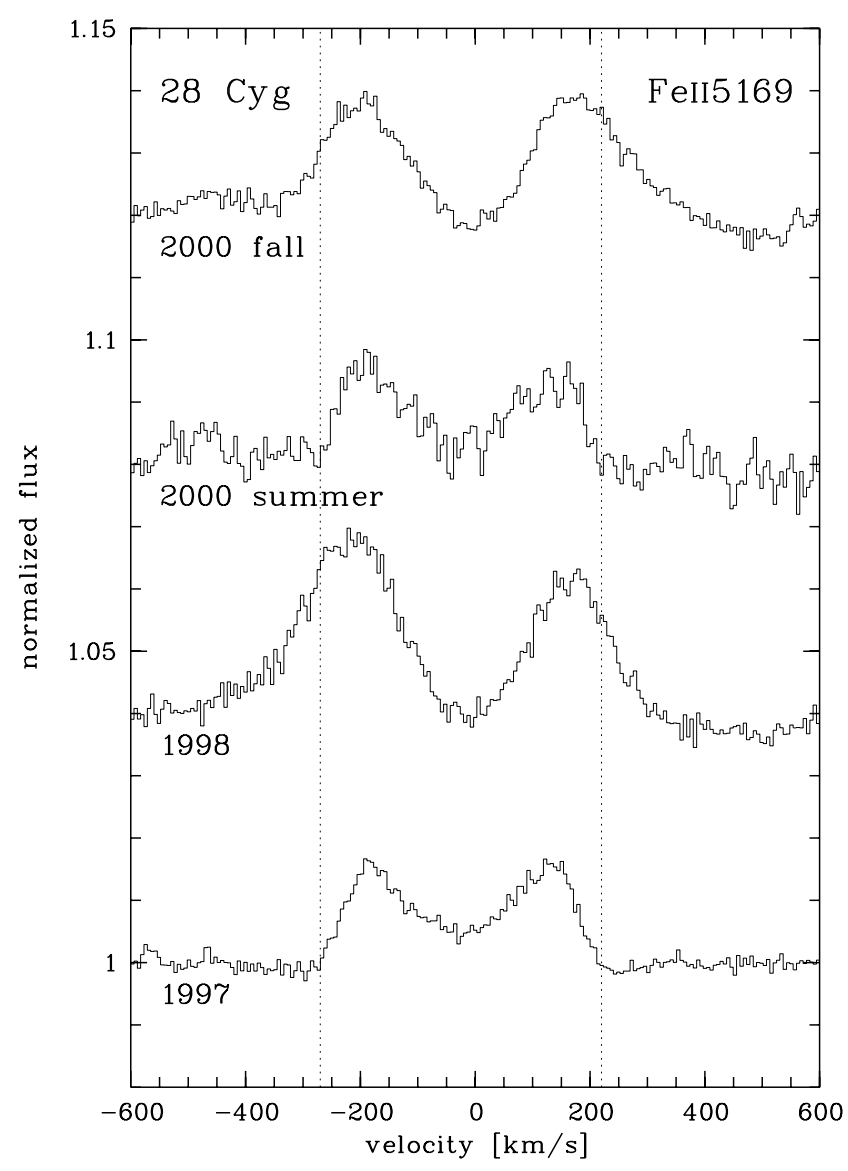

Fig. 7. The appearance of the Fe II 5169 line of 28 Cyg in the average spectra of four different observing campaigns. The dotted lines represent the narrowest appearance of the Fe II 5169 emission of $[-270,220] \mathrm{km} \mathrm{s}^{-1}$. The disappearance of the high velocity tails in the emission, while the narrower component remains largely unchanged, is in agreement with the hypothesis that only the emission contribution from the inner parts of the disk is gone.

- especially in optically thick lines, the variability of the base width is very little connected to that of the main peaks, which may even remain nearly constant. In particular, the waxing and waning of emission line wings is a genuine variability. That is, it is not due to the homologous growing and fading of emission profiles during the course of which the wings would be pushed above or below some detection threshold.

Since variability of the base width of emission lines is an integral part of outbursts, this list supplements the temporal profile of outbursts given in the Introduction.

In $\mu$ Cen (Rivinius et al. 1998a), and possibly also 28 Cyg (Tubbesing et al. 2000), outbursts repeat with high regularity. Cyclic photometric outbursts were detected by Hipparcos (Hubert \& Floquet 1998) in a still larger sample of Be stars. For these reasons, this study adopts the concept of (cyclically) repeating mass loss events (outbursts) as a working hypothesis for the underlying process of the above described variability of all stars in the present sample.

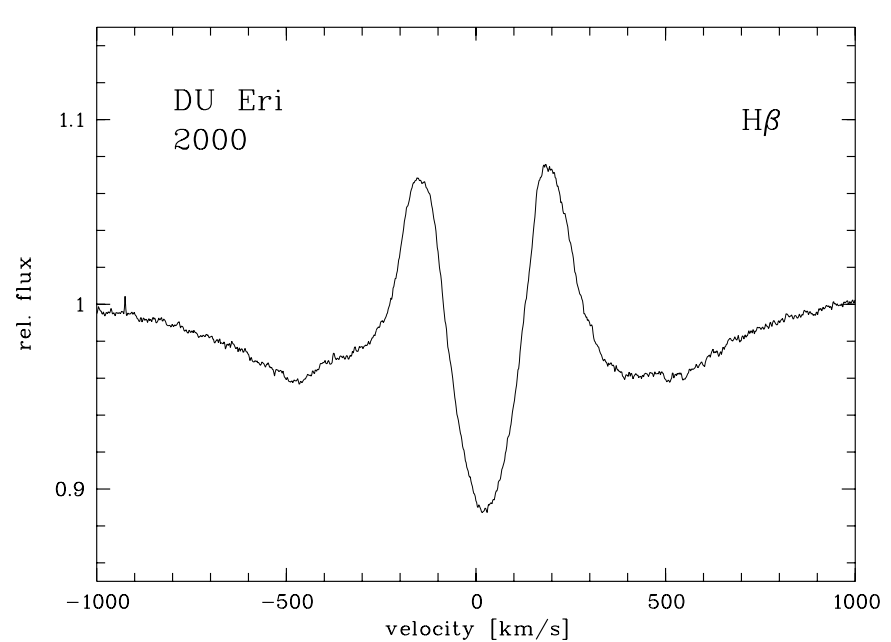

Fig. 8. The $\mathrm{H} \beta$ line of DU Eri in January 2000. The unusual morphology of the emission wings is reminiscent to FV CMa, but the metallic emission is too weak (i.e. below the detection threshold) to provide further evidence from optically thin lines.

Considering only the variability of the base width of emission lines, FV CMa would not qualify for inclusion in the present sample because it was only observed for a short time. However, the similarity of these spectra to $28 \mathrm{CMa}$ and especially X Per, in which variable emission line wings not only exist but temporarily took on the same shape of a second pair of discrete emission peaks with larger separation, makes it a strong candidate. DU Eri is a comparable case.

Outbursts repeat on time scales between 1-2 months ( $\mu$ Cen) and a few years. The time scale for the decay of enhanced emission line wings depends on the magnitude of the outburst but typically is of the order of a few months. Disks of Be stars also undergo still longer-term variations, which may take up to several decades. They mainly consist of changes of the emission peak height and are probably related to changes of the (outer) dimensions and the total mass content of the disk. Since they are not sufficiently sampled by the present observations, the remainder of the paper ignores such long-term trends. Similarly, because all stars in this sample exhibit symmetric emission profiles, global disk oscillations, which observationally reveal themselves by cyclic long-term $V / R$ variations, are also not considered.

\section{Disk structure}

Be star disks have succesfully been modeled as relatively thin, quasi-Keplerian disks. The Keplerian approximation gives a disk of increasing opening angle with radius, which is typically assumed to be isothermal. The density law in the disk is commonly derived with the help of the equation of continuity, the assumption of constant base density $\rho_{0}$, and a constant outflow speed. Due to the outburst activity, however, $\rho_{0}$ is certainly variable, and hence the disk density structure becomes a free parameter, while all other properties should remain unchanged in first order approximation. Therefore, the ring, as proposed in this paper, can 
be imagined as a Keplerian disk, in which the innermost part is missing.

\subsection{Inner disk radius}

Numerous authors have investigated which conclusions about the disk structure can be drawn from observed emission line profiles (e.g. Poeckert \& Marlborough 1978; Dachs et al. 1986; Hummel \& Dachs 1992; Hummel 1994; van Kerkwijk et al. 1995). The results show quite clearly that deriving robust results in terms of uniqueness of the obtained parameters is almost impossible by inspecting snap-shot emission line profiles.

On the other hand, the qualitative interpretation of variations of a given disk is much less dependent on the particulars of the correct model. For instance, $\sin i$ is the same for all observations, and therefore the effect of the corresponding uncertainty in the interpretation is much reduced. Moreover if lines from different ions and transitions were observed simultaneously, the need to accommodate them in a single, self-consistent description provides very strong constraints.

To the first order, two circumstances are pertinent to the interpretation of the variable base width of emission lines in the present sample of Be stars:

- Virtually all currently-proposed disk models include a rotational velocity profile decreasing monotonically with radius. As briefly summarized in Sect. 1, there is even some evidence that the rotational velocity law is crudely Keplerian. Since large-scale radial motions are very much slower, emission from the inner regions of the disk, where the velocities are the largest, will appear in the outermost line wings. E.g. for a Keplerian disk with circular orbits, the maximum projected velocity at a given radius is

$$
\Delta v_{\text {edge }}=2 v_{\text {kepl }} \sin i\left(\frac{R_{\text {peak }}}{R_{\star}}\right)^{-\frac{1}{2}}
$$

- If a given disk can be assumed to be in quasiequilibrium (i.e. radiatively, thermally, hydrodynamically, and at constant inclination angle; see Sect. 4 for a discussion of the last assumption) the only physical parameter that can change the emission formed at a given radius is the gas density.

The combination of these two arguments implies that the variability of the emission base width is due to density variations in the inner disk. Since the remainder of the emission profiles often is much less affected (and/or on much longer time scales), this leads to the notion of the development of an inner cavity at times when the extreme wings disappear or significantly weaken. The remaining disk structure would be more reminiscent of a detached ring.

In mosts stars, also the separation of the emission peaks is variable. However, this quantity traces the radius of maximum emissivity of the disk for a given transition and should not be confused with the emission base width. Although changes at the inner edge of the disk, changing the base width, will typically influence the emission peak separation in the same sense (as seen e.g. in FW CMa or $\omega \mathrm{CMa}$ ), this correlation might be weak or even absent in some cases, since it also depends on the properties of the outer disk regions.

Optically thick lines need to be treated with more caution because the most extreme emission line wings must be suspected to contain a large scattering component (Poeckert \& Marlborough 1978). Therefore, the observed maximal velocities in lines like $\mathrm{H} \alpha$ are not neccesarily indicative of the (rotation dominated) ion kinematics, but rather of the (temperature dominated) electron kinematics.

In the absence of such a complication, optically thick lines can be conveniently used to illustrate a few more details, because the resulting emission line profiles depend at each line-of-sight velocity only on the surface area having this velocity. By contrast to optically thick ones, optically thin lines have to be volume-integrated, and their region of formation is typically close to the star. For Balmer lines, in turn, one can safely assume they form almost throughout the entire disk. Therefore, when an inner cavity develops in a purely Keplerian disk, optically thick emission lines (Owocki 2001, private communication)

- suffer the smaller relative flux changes in their wings the larger the disk is, because the area having such projected velocities becomes smaller in comparison;

- retain the position of their peaks because the latter originate from the outermost regions of the disk, which have the largest surface area in a given velocity interval.

Since the observed variability of optically thick lines like $\mathrm{H} \alpha$ can indeed be described in such terms (see Fig. 4, for instance), the above inference of a variable inner disk radius, derived from optically thin lines, is also in agreement with data from optically thick lines.

The photometric observations of outburst behaviour (Hubert \& Floquet 1998, brightenings and fadings) are well explainable in terms both of a variable cavity, and a solid disk of variable mass, since only the innermost part of the disk contributes significantly to the continuum flux in any case. Simultaneous spectra could discriminate between these two options, but do not seem to exist.

\subsection{Multiple disks}

In $\omega$ CMa a second, morphologically distinct emission constituent was observed in 2000 while the previous one was still partly preserved (Fig. 1). The high velocities, if due to rotation, imply that it arises from matter close to the star. The dual structure of the O I 8446 emission profile without a smooth transition between the broad base and the inner peaks, as well as the evolution of the Fe II and Si II emission (Fig. 1), support the notion that the newly ejected gas and the remainders of the old disk are not spatially connected. The outburst in 2000 possibly filled up (part of) a circumstellar low-density region, which may previously have formed in the old disk. 
Although there are no observations of other lines at the same epoch, the $\mathrm{H} \alpha$ profile observed in 1992 by Hanuschik et al. (1996) qualifies DU Eri as a second candidate star, whose circumstellar envelope temporarily consisted of an inner disk and an outer, presumably older, ring. In January 2000, a similar structure was observed (Fig. 8), but not as clearly as in 1992, and no metallic emission lines could be detected for further comparison.

A still stronger case in support of the dual constituents picture is represented by FV CMa. Unlike in $\omega \mathrm{CMa}$, a broad base in the O I 8446 emission can hardly be seen in this star because, owing to much stronger kinematical broadening, the line is blended with $\mathrm{Pa}_{14}$. However, the similarity of $\mathrm{O}$ I 8446 in $\omega \mathrm{CMa}$ to the $\mathrm{H} \beta$ emission of FV CMa is striking (Fig. 2, residuals, vs. Fig. 1, O I 8446, uppermost spectrum).

In fact, the Balmer line profiles include two sets of edges, which bracket the velocity ranges $[-170,+245] \mathrm{km} \mathrm{s}^{-1}$ and $[-300,+345] \mathrm{km} \mathrm{s}^{-1}$, respectively. Starting with $\mathrm{H} \gamma$, the range between the two blue and the two red edges $([-300 \ldots-170$ and $+245 \ldots+345] \mathrm{km} \mathrm{s}^{-1}$ ) takes on the appearance of a secondary double-peaked emission constituent.

Because Balmer emission lines are easily distorted by scattering processes (Poeckert \& Marlborough 1978), the translation of line widths into disk velocities and spatial dimensions is not straightforward. This affects $\mathrm{H} \alpha$ most strongly and becomes less important for the higher Balmer lines. However, in the case of FV CMa the sharpness of the edges, their equal position in all Balmer lines, and the even increasing prominence of the edges towards bluer Balmer lines (cf. Fig. 2) render a geometric interpretation of these values a plausible approach. This is supported by the higher Paschen lines, which are morphologically intermediate between $\mathrm{H} \gamma$ and $\mathrm{H} \beta$, as well as by the He I and metallic lines displaying emission components. The HeI5876, 6678, 7065 and Si II 6347 lines show clear emission up to $[-300,+370] \mathrm{km} \mathrm{s}^{-1}$. This indicates that these are real gas velocities rather than scattering broadening, since scattering broadening does not produce a sharply defined egde of the emission, as seen in these lines. Lines like Fe II 5169 have the edges $[-170,+245] \mathrm{km} \mathrm{s}^{-1}$ in common with the Balmer lines. But their broad emission constituent does not extend over the full range of $[-300,+370] \mathrm{km} \mathrm{s}^{-1}$.

Application of the relation between projected rotational velocity and inner disk radius (Sect. 3.1) and the assumption that the broad emission component belongs to a disk starting right above the stellar surface $\left(2 v_{\text {kepl }} \sin i=\right.$ $670 \mathrm{~km} \mathrm{~s}^{-1}$ ) imply that the narrow component originates from a ring with an inner radius of at least $2.6 R_{\star}$. This number will be higher still if the inner disk also is detached from the star. However, this is not too likely as it would require an even higher Keplerian velocity at the stellar surface.

Furthermore, the difference in the Balmer decrement of both constituents in FV CMa is indicative of a higher density in the inner disk than in the outer ring.
Note also that a disk with two different constituents of different radii has been postulated before by Hanuschik et al. (1988) on account of the inflection points seen in $\mathrm{H} \alpha$ emission lines such as those of $\omega \mathrm{CMa}$ and $\mu$ Cen (because of their appearance also dubbed winebottle profiles). However, Hummel (1998a) later showed that a smooth radial run of the ratio of the local line broadening by gas motions to non-coherent scattering reproduces such profiles of optically thick lines quite satisfactorily.

\subsection{Disk evolution}

The above static description can be easily extended to the notion that after an outburst the disk begins directly above the stellar surface and later a slowly outwards growing inner cavity develops. The more time this cavity has to grow, i.e. the longer the intervals between (major) outbursts are, the more distinct the ejecta of a later outburst should be from the ring in distance from the star, velocity, and, therefore, the emission line profiles. The combination of the changes in the emission profile morphology and width, observed best in $\mu$ Cen and $\omega \mathrm{CMa}$, supports this view well. The times between outbursts range from weeks in $\mu$ Cen to perhaps as much as years in $\omega$ CMa and FW CMa. Hubert \& Floquet (1998) report characteristic times between outbursts of $\omega \mathrm{CMa}$ of 200 and 330 days. The sampling of the present observations would not be sensitive to them. But the data suggest that major outbursts occurred only in 1996 and 2000. Something similar may be true of FW CMa.

In $\eta$ Cen (Sect. 2.1) the total base width of the He I 6678 emission was observed to be $680 \mathrm{~km} \mathrm{~s}^{-1}$ before and $1100 \mathrm{~km} \mathrm{~s}^{-1}$ after the outburst, respectively. About two months later, the total base width had decreased to $660 \mathrm{~km} \mathrm{~s}^{-1}$ again. Using $\sin i=1$ and $v_{\text {crit }}=600 \mathrm{~km} \mathrm{~s}^{-1}$ (see Rivinius et al. 1999, for a justification of these numbers and the equations used), the sudden change of the inner disk radius during the outburst can be estimated. The inner radius of the HeI 6678 emitting region was $3.1 R_{\star}$ shortly before the outburst. Directly thereafter this number was only $1.2 R_{\star}$. Note that this value did not decrease smoothly, but changed rather suddenly in the wake of a discrete mass loss event. After two months, the inner edge radius had slowly grown again to $3.3 R_{\star}$. The star was not observed for two weeks, but afterwards it was clear that again an outburst must have occurred in the meantime, since all the velocity separations again were high.

Observational constraints can also be placed on the evolution of the outer disk radius. For example, Rivinius et al. (1999) (their Sect. 5.3) used a morphological line profile feature specific to shell stars, a so-called central quasi emission (CQE), to probe the disk structure of $\eta$ Cen after an outburst. They found that in the weeks after the outburst the outer disk radius only grew from 6.2 to $6.8 R_{\star}$ (deduced from the CQE in He I 6678) or 7.3 to $8.7 R_{\star}$ (derived from the $\mathrm{H} \alpha$ emission peak separation). At the same time the radius estimated from the He I 6678 
Table 1. Evolution of inner disk radius, emission peak radii (representative of the bulk of the emitting material) and outer disk radius before, during, and after an outburst in $\eta$ Cen in February 1996. For explanation of methods and symbols see Sect. 3.3. All dimensions are given in units of the equatorial stellar radius.

\begin{tabular}{l|ccc|c}
\hline $\begin{array}{l}\text { Modified } \\
\text { Julian } \\
\text { date }\end{array}$ & $\begin{array}{c}|c| \\
\text { inner edge } \\
v_{\max }\end{array}$ & $\begin{array}{c}\text { bulk } \\
v_{\text {peak }}\end{array}$ & $\begin{array}{c}\mathrm{H} \alpha \\
\text { CQE }\end{array}$ & $\begin{array}{c}\text { Culk } \\
v_{\text {peak }}\end{array}$ \\
\hline $50101-119$ & 3.1 & 6.0 & 7.1 & 9.0 \\
$50121-126$ & 1.2 & 2.8 & 6.2 & 7.3 \\
$50199-204$ & 3.3 & 4.8 & 6.8 & 8.7 \\
\hline
\end{tabular}

emission peaks, which are formed much closer to the star than both the CQE and the $\mathrm{H} \alpha$ emission, almost doubled from 2.8 to $4.8 R_{\star}$.

These numbers, summarized in Table 1 and sketched in Fig. 9, are based on several simplifications (e.g., $\sin i=1$, estimated $v_{\text {crit }}$ ) so that their absolute values must be treated with care. But the qualitative conclusion of a sudden decrease of the inner disk radius during an outburst, and the subsequent slower, smooth increase is robust. Combined with the accompanying much smaller increase of the outer disk dimensions this justifies the picture in which an inner low density region forms a while after an outburst, the next outburst fills it up again, and finally this new material possibly merges with the older ring farther out.

For the explanation of their observations of X Per, Roche et al. (2000) have independently proposed a multiple-disk structure. Because of the other stars under consideration only o And is a known binary, it appears plausible that the variability at the inner edge of the disk of this system is unrelated to the presence of a companion. Neutron stars orbiting Be stars have for a long time been realized to be promising probes of the disk structure. The present observations suggest this even more strongly. But they also imply that the corresponding X-ray monitoring must be accompanied by a series of high-quality observations of emission line profiles, which need to start weeks to months earlier.

\section{Discussion}

\subsection{Alternative hypotheses}

For disks of the kind considered here, there is only one other plausible general mechanism to explain a variable base width of the optically thin emission, namely a varying tilt angle of the disk (Hummel 1998b). However, for random orientations with regard to the Earth, one would expect either that increases and decreases in line emission and base width in a given star take the same time or that sudden increases followed by slow decreases are roughly as often observed as the inverse (slow increase followed by sudden decrease). The present database is at variance with both. Also the change of the base width at roughly constant emission peak separation (Fig. 7) cannot be explained by varying tilt angles.

For several stars discussed above, circumstances specific to them or a particular event may as well lead to similar observational effects. Examples are:

- In FV CMa the temporarily enhanced emission wings in the Balmer lines could be caused by a transient local overdensity. However, this would not explain that in stars like $\omega \mathrm{CMa}$ the emission lines of Fe II and Si II vanish completely and return only after a new burst when also the broad wing component in Balmer lines appears again. Nor can it account for the variable base width of the optically thin emission lines.

Also, in those cases where the development of the emission lines after an outburst could be observed in sufficient detail, the temporary emission at high velocities always decreased monotonically and roughly homologously with time. Therefore, the simultaneous presence of two distinct emission constituents as in FV CMa is also for this reason much more plausibly attributed to two outbursts at different times than to a radially outward moving density enhancement, superimposed on an otherwise unchanged disk. This, in turn, re-inforces the inference of a transient inner low-density region;

- Moderate increases in the base width of optically thin lines by less than about $20 \mathrm{~km} \mathrm{~s}^{-1}$, like in FW CMa, might be explainable by variable turbulence. But the difference in base width of more than $200 \mathrm{~km} \mathrm{~s}^{-1}$ observed in 28 Cyg (Tubbesing et al. 2000, and Fig. 7) certainly cannot be attributed to this effect.

But it would be surprising if such very different mechanisms would lead to the rather homogeneous variability patterns observed in the present sample of stars.

\subsection{Synopsis of the formation of inner disk cavities with physical processes}

The present observations do not offer direct evidence as to whether the matter leaving the inner disk falls back on to the star or moves to larger distances. It is quite probable, however, that a disk cannot persist for a long time without on-going star-to-disk mass transfer:

- Viscous decretion, which succeeds in explaining more observational details of Be star disks than most other models (for a discussion see Porter 1999; Okazaki 2001) by necessity leads to an outflow;

- Radiation pressure alone is insufficient to overcome gravity at the photospheric level, except for the earliest B spectral subtypes. Even in these, however, the observed (and expected) winds are weaker than observed in most Be stars. But the stronger, possibly even full, rotational support of matter in the disk may give it much more leverage there.

The coming and going of Balmer line emission in $\mu$ Cen, when it did not have a persistent disk (Baade et al. 1988; Hanuschik et al. 1993), probably provides the observational confirmation. 

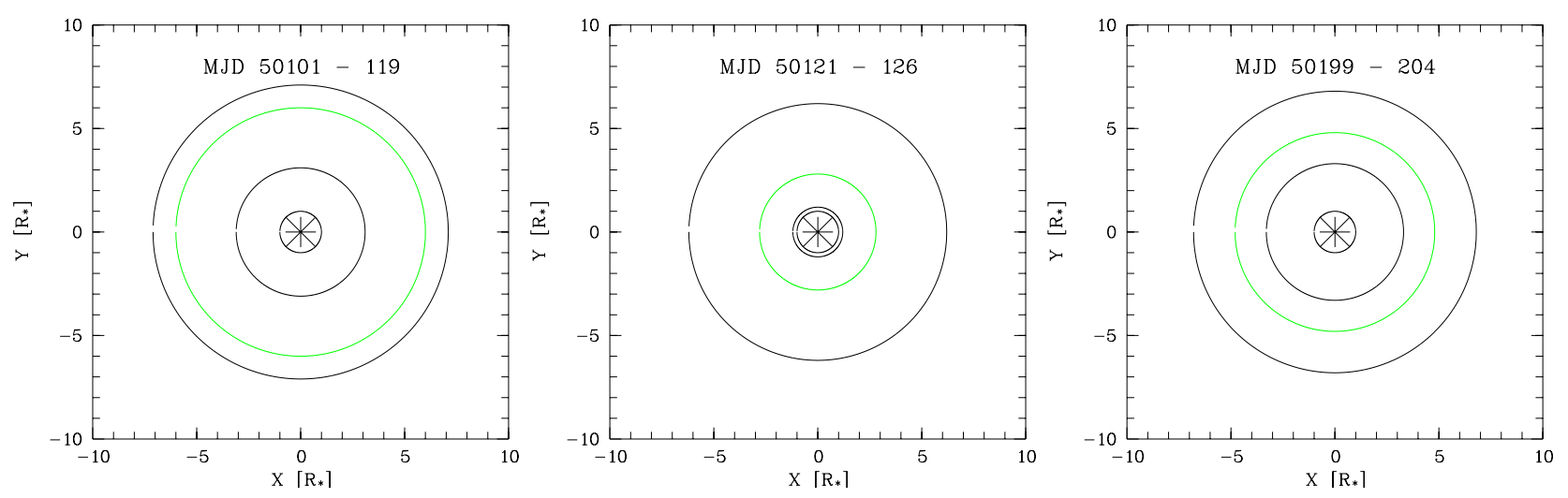

Fig. 9. Sketch of the disk structure of $\eta$ Cen shortly before, after, and two months after an outburst, just before the subsequent one. The star is indicated by the crossed circle, the dark circles represent the inner and outer edges of the disk, the gray line the main bulk of emitting material. The plots base on He I6678 data, as described in Table 1 and Sect. 3.3.

Outward motions in a (pseudo-)Keplerian disk pose the well-known angular momentum problem, which makes it difficult to explain the very existence of such disks. In numerical simulations, Kroll \& Hanuschik (1997) studied the trajectories of particles ejected from a rapidly rotating star in explosive events. They found that a collisional exchange of angular momentum takes place, which permits some particles to settle in an orbit and forces others to fall back to the star. Conceivably, radiative and/or viscous perturbations of the inner edge of the disk lead to processes with a similar effect. Moreover, in the viscous decretion model, the disk is not Keplerian everywhere but probably becomes angular momentum conserving in its outer parts (Okazaki 2001), thereby alleviating the problem.

In order for viscous decretion disks to survive for many decades, the mass supply to the disk needs to be either continuous or composed of discrete events with a repetition time significantly shorter than the viscous timescale (cf. Okazaki 2001). The outburst periods of 29 and 54 days in $\mu$ Cen (Rivinius et al. 1998b) satisfy this criterion easily. In the Hipparcos database, Hubert \& Floquet (1998) identified 14 candidate stars with photometric cycle lengths between 20 and 500 days. This is still considerably shorter than the viscous timescale of typically 5 years.

Model calculations by Gayley et al. (2001) and mainly aimed at explaining long-term, precession-like variations of the overall structure of Be star disks provide a first numerical description of the dynamical influence of the stellar radiation on a Keplerian disk. Because of the shadowing effects of spectral lines, the radiative driving of a gas outflow requires the presence of velocity differences in order to be able to further accelerate the gas. Therefore, the radiation from a central point source would have no effect on a Keplerian disk because the velocity profile is flat in the radially outward direction. Only the finite angle subtended by the stellar photosphere at each location in the disk provides the required velocity differences required.
This model would be most effective in the case of lowdensity disks while the self-shadowing of lines in highdensity disks could even let it fail. In fact, a sufficiently high density at the beginning of the formation of a disk might be required for the survival of the disk (leading to the interesting question of whether early-type Be stars can develop a disk only because of their outbursts). If the same process is also involved in the later dispersal of a disk, some further ingredients are probably necessary for the full explanation.

Finally, a very effective circumstellar broom as well as angular momentum booster could obviously be made of magnetic field loops. They could be small and weak enough so as not to be detectable with current instruments, so that observational constraints would be difficult to supply.

\subsection{Implications for the modeling of disks}

The observations presented in this paper call for timedependent refinements of the models of Be star disks at the interface to the star (as also noted by Okazaki 2001). Even in the absence of multi-constituent structures as in FV CMa, the inner disk radius should be treated as a free parameter. It may be possible that only after an outburst is there no significant density minimum between photosphere and disk. However, during these early stages the validity of the assumption of equilibrium becomes questionable.

If the described variability of the inner disk regions is indeed due to outbursts of the central star, it is probably essential to keep in mind that outbursts do not seem to be common, or may even be absent, in late-type Be stars. With the exception of $o$ And, all stars of the present sample have spectral types between B1 and B3 (as have the 14 HipparCos stars [Sect. 4.2]). Therefore, the results of this work may well not be pertinent to the disks of Be stars 
of all spectral types. On the other hand, there is mounting evidence that among the early sub-types outbursts are very common.

The inner variability appears to have little to no effect on the outer disk regions. Typically observed outflow velocities in Be star disks are only a few $\mathrm{km} \mathrm{s}^{-1}$ (e.g. Rivinius et al. 1999). This applies to the ring. Closer to the star, the (radiative?) sweeping of the inner cavity is more effective. In this way the ring forms a reservoir, from which the flux of matter in the outer disk regions is fed. Unless such a reservoir empties significantly, it has a damping effect on the amplitudes of pertubations propagating through it.

Therefore, the density power laws derived from infrared and radio data using the equation of continuity (Waters 1986) should describe these regions well, as the assumption of a constant mass flow might be a good approximation for the outwards dissipation of the ring, even if the feeding from inside is due to an eruptive process.

\subsection{Disks and winds}

For more than a decade, observations with the International Ultraviolet Explorer (IUE) satellite have been one of the governing factors in the research of $\mathrm{Be}$ stars. However, much of this work was dominated by an intensive dispute about the geometry of the exophotospheric regions: Do (i) the optical low-velocity circumstellar emission and absorption lines due to lowly ionized gas and (ii) the high-velocity lines from highly ionized species found by IUE evidence a radial stratification similar to the solar photosphere and chromosphere? Or do the former form in an equatorial disk and the latter in a polar wind? A first access to the vast amount of observations and interpretations can be obtained through the monograph by Underhill \& Doazan (1982) and the literature cited therein.

Now that the observations no longer need to explain the geometry but the known geometry can help to better understand the observations, it is worthwhile to briefly revisit some of the loose ends. The incipient glimpses of the life cycles of disks add an extra component. The following points come to mind:

Polar winds: advocates of the flat-disk model resorted to the hypothesis that the fast wind filled the space above and below the disk. The notion of a polar wind also seems to be helped by the decrease in effective temperature (and, thereby, radiative driving) from pole to equator as a result of rotationally-induced gravity darkening.

However, the key study based on the largest sample of Be star spectra obtained with IUE does not support this at all: Grady et al. (1987a, 1989) found that in Be stars with $v \sin i$ less than about $150 \mathrm{~km} \mathrm{~s}^{-1}$ the winds were not any stronger than in ordinary B stars. The authors surmised that there might be Be stars which rotate too slowly to develop a wind. But this distinction is not supported by any other circumstance. If, conversely, it is assumed that (early-type) Be stars do form a reasonably homogeneous group, the conclusion must be that the wind excess of Be stars over B stars forms neither at the pole nor very close to the star: it is the result of ablation of the disk by the stellar radiation.

Wind/disk correlations: both wind and disk are highly variable in many Be stars. The lack of a clear correlation was used as a supporting argument by the disk camp, whereas the other one claimed superiority because the variations were not completely uncorrelated. The notion of a disk being created, or replenished, by outbursts and then being gradually dispersed can explain such a loose relation without any effort.

Most importantly, neither of the two historicallycompeting models would be able to explain observations like those of $\theta \mathrm{CrB}$ : after a Be-shell phase, the star entered a (still ongoing) B star phase between March und October 1980, i.e. no trace of circumstellar material was observed since October 1980 in the visual domain. Yet, the IUE observations showed shell lines in e.g. Si IV 1394, Al III 1863, and Fe III 1896 until about December 1981 (Doazan et al. 1986b) and a continuous wind excess in C IV 1548 with relatively low expansion velocities until March 1982 (Doazan et al. 1984, 1986a).

Now, if the excess wind is merely the result of the demolition of the disk rather than its formation, this is unavoidable because the much larger optical thickness of the UV resonance lines makes them still visible when the column density is far too low for Balmer and optical metal lines.

After March 1982, decreasingly frequent signs of a temporarily enhanced wind were observed at several occasions in $\theta \mathrm{CrB}$, having higher expansion velocities than before (Doazan et al. 1987). These might have been indications of failed disk formation, as sketched at the end of Sect. 4.2.

Such a scenario can be easily adapted to explain observations of $\lambda$ Eri and 66 Oph (Barker \& Marlborough 1985; Barker 1986; Grady et al. 1987b; Peters 1988), if it is assumed that the excess wind is strongest during the sweeping of the inner ring cavity, i.e. after an outburst.

Discrete Absorption Components (DACs): at one time or another, DACs are observed superimposed on the wind lines of virtually all luminous OB stars (Howarth \& Prinja 1989). Massive observing campaigns with IUE found them to repeat cyclically, and the commonlyaccepted explanation is by shocks arising at so-called co-rotating interaction regions (CIRs). In some stars, there is also evidence of CIRs developing due to stellar nonradial pulsation (Howarth et al. 1998).

The same models are undoubtedly also applicable to Be stars. However, in Be stars DACs account for a very much larger fraction of the equivalent width of 
UV wind lines than in more luminous OB stars, and on average also appear to occur more often. Moreover, at least some fractions of the DACs in Be stars do not seem to repeat cyclically. This has led some authors to infer special physical conditions in the atmospheres of Be stars. But it is rather probable that the gradual destruction of the disk, and the dynamical response of the disk matter to this process, do not proceed in a smooth and continuous fashion. It is, therefore, conceivable that the different roles of DACs in Be and more luminous OB stars are only the result of the presence of a decaying disk.

The work of Telting \& Kaper (1994) might support such a hypothesis, as they found the occurence of DACs in $\gamma$ Cas strongly correlated with the $V / R$ ratio of the disk's Balmer emission. They concluded that the material forming the DACs comes from the density wave rather than from the star, leaving the disk outwards on a trajectory resembling the case of conservation of angular momentum. Such a scenario would also be compatible with the model by Okazaki (2001).

Very rapid variability: variations within hours mainly concern DACs. Therefore, the same conjectures as before suggest the same change in paradigm: The combination of wind and disk instabilities may well make the assumption of special physical processes in Be stars unnecessary.

\section{Conclusion}

It seems necessary to adjust somewhat the picture of the disks around early-type Be stars. Instead of a stationary disk structure with constant, moderate outflow, several observed cases show indications for a varying radius of the inner disk edge, i.e. at times forming a ring.

The appearance of a disk in contact with the stellar surface seems limited to the immediate times of outbursts. Due to a process yet to be identified, part of the ejected matter attains sufficiently high angular momentum to form a roughly Keplerian disk (for instance, this may be due to the collisional exchange of angular momentum between particles, in which case the angular momentum donors would return to the star).

After a dynamically stable disk has formed, intrinsic instabilities of the disk, starting at the inner edge (e.g., due to sheer), and the stellar radiation pressure gradually excavate the disk, which may be re-filled by subsequent outbursts. Simultaneous with the growth of the inner disk radius, measurements of the mean and outer disk radius also indicate expansion. All these properties typically follow a saw-tooth pattern, steeply changing during outburst and gradually returning afterwards.

The excavation of the inner disk, eventually turning into a gradual erosion of the entire disk (if no further, sufficiently strong outbursts take place: until its complete dissolution), accounts for the differences between the winds of Be and B stars.
The direct observations of a varying inner disk radius are limited to the cases described in this paper. However, not only are several other, but less conclusive indicators seen more frequently (e.g. varying peak seperation), but the evolution from a disk into a ring is closely correlated with circumstellar outbursts, which are common for earlytype Be stars. Therefore, it seems plausible to assume that the mechanisms at work to form a ring are also effective in other, if not all, early-type Be stars.

Acknowledgements. This work was supported by the Deutsche Forschungsgemeinschaft (436 TSE 113/18), the Academy of Sciences of the Czech Republic (436 TSE 113/18), and the Grant Agency of the Academy of Sciences of the Czech Republic (A3003001). We are grateful to the Feros Consortium (PI: Bernhard Wolf) for the opportunity to make use of a fraction of their guaranteed observing time in June, 1999. Reinhard Hanuschik and Wolfgang Hummel raised numerous interesting points in joint discussions that helped to improve the manuscript. We are indebted to Stan Owocki for enlightening suggestions for the presentation of the paper and the discussion of the results.

\section{References}

Baade, D. 1982, A\&A, 105, 65

Baade, D. 2000, in The Be Phenomenon in Early Type Stars, ed. M. A. Smith, H. F. Henrichs, \& J. Fabregat, ASP Conf. Ser., 214, IAU Colloq., 175, 178

Baade, D., Dachs, J., van de Weygaert, R., \& Steeman, F. 1988, A\&A, 198, 211

Balona, L. A. 1999, MNRAS, 306, 407

Barker, P. 1986, PASP, 98, 44

Barker, P. K., \& Marlborough, J. M. 1985, ApJ, 288, 329

Bjorkman, J. 2000, in The Be Phenomenon in Early Type Stars, ed. M. A. Smith, H. F. Henrichs, \& J. Fabregat, ASP Conf. Ser., 214, IAU Colloq., 175, 435

Dachs, J., Hanuschik, R., Kaiser, D., \& Rohe, D. 1986, A\&A, 159,276

Delgado-Martí, H., Levine, A. M., Pfahl, E., \& Rappaport, S. A. 2001, ApJ, 546, 455

Doazan, V., Marlborough, J. M., Morossi, C., et al. 1986a, A\&A, 158, 1

Doazan, V., Morossi, C., Stalio, R., \& Thomas, R. N. 1986b, A\&A, 170, 77

Doazan, V., Morossi, C., Stalio, R., Thomas, R. N., \& Willis, A. 1984, A\&A, 131, 210

Doazan, V., Rusconi, L., Sedmak, G., \& Thomas, R. N. 1987, A\&A, 173, L8

Gayley, K. G., Ignace, R., \& Owocki, S. 2001, ApJ, submitted

Grady, C. A., Bjorkman, K. S., \& Snow, T. P. 1987a, ApJ, 320,376

Grady, C. A., Bjorkman, K. S., Snow, T. P., et al. 1989, ApJ, 339, 403

Grady, C. A., Sonneborn, G., Wu, C., \& Henrichs, H. F. 1987b, ApJS, 65, 673

Hanuschik, R. W. 1996, A\&A, 308, 170

Hanuschik, R. W., Dachs, J., Baudzus, M., \& Thimm, G. 1993, A\&A, 274, 356

Hanuschik, R. W., Hummel, W., Sutorius, E., Dietle, O., \& Thimm, G. 1996, A\&AS, 116, 309 
Hanuschik, R. W., Kozok, J. R., \& Kaiser, D. 1988, A\&A, 189, 147

Hazlehurst, J. 1967, Z. Astrophys., 65, 311

Howarth, I. D., \& Prinja, R. K. 1989, ApJS, 69, 527

Howarth, I. D., Townsend, R. H. D., Clayton, M. J., et al. 1998, MNRAS, 296, 949

Huang, S. S. 1972, ApJ, 171, 549

Hubert, A. M., \& Floquet, M. 1998, A\&A, 335, 565

Hummel, W. 1994, A\&A, 289, 458

Hummel, W. 1998a, A\&A, 289, 458

Hummel, W. 1998b, A\&A, 330, 243

Hummel, W., \& Dachs, J. 1992, A\&A, 262, L17

Kaufer, A., Stahl, O., Tubbesing, S., et al. 1999, ESO Messenger, 95, 8

Kroll, P., \& Hanuschik, R. W. 1997, in Accretion Phenomena and Related Outflows, ASP Conf. Ser., 121, IAU Colloq., 163,494

Limber, D. N., \& Marlborough, J. M. 1968, ApJ, 152, 181

Maintz, M., Rivinius, Th., Tubbesing, S., et al. 2000, in The Be Phenomenon in Early Type Stars, ed. M. A. Smith, H. F. Henrichs, \& J. Fabregat, ASP Conf. Ser., 214, IAU Colloq., 175,244

Millar, C. E., \& Marlborough, J. M. 1999, ApJ, 526, 400

Okazaki, A. 2000, in The Be Phenomenon in Early Type Stars, ed. M. A. Smith, H. F. Henrichs, \& J. Fabregat, ASP Conf. Ser., 214, IAU Colloq., 175, 409

Okazaki, A. T. 2001, PASJ, 53, 119

Peters, G. J. 1988, PASP, 100, 207

Poeckert, R., \& Marlborough, J. M. 1978, ApJS, 38, 229

Porter, J. M. 1999, A\&A, 348, 512

Quirrenbach, A., Bjorkman, K. S., Bjorkman, J. E., et al. 1997, ApJ, 479, 477

Rivinius, Th. 1999, in Variable and Non-spherical Stellar Winds in Luminous Hot Stars, ed. B. Wolf, O. Stahl, \& A. W. Fullerton (Springer), Lect. Notes Phys., IAU Colloq., 169, 36

Rivinius, Th., Baade, D., Štefl, S., et al. 1998a, A\&A, 333, 125

Rivinius, Th., Baade, D., Štefl, S., et al. 1998b, in Cyclical variability in stellar winds, ed. L. Kaper, \& A. Fullerton, ESO Conf. Ser., 207
Rivinius, Th., Štefl, S., \& Baade, D. 1999, A\&A, 348, 831

Roche, P., Tarasov, A. E., Lyuty, V. M., Clark, J. S., \& Larionov, V. 2000, in The Be Phenomenon in Early Type Stars, ed. M. A. Smith, H. F. Henrichs, \& J. Fabregat, ASP Conf. Ser., 214, IAU Colloq., 175, 589

Slettebak, A. 1982, ApJS, 50, 55

Smith, M. A., Peters, G. J., \& Grady, C. A. 1991, ApJ, 367, 302

Štefl, S. 1999, in Variable and Non-spherical Stellar Winds in Luminous Hot Stars, ed. B. Wolf, O. Stahl, \& A. W. Fullerton (Springer), Lect. Notes Phys., IAU Colloq., 169, 24

Štefl, S., Baade, D., Rivinius, Th., Stahl, O., Wolf, B., \& Kaufer, A. 1998, in A Half Century of Stellar Pulsation Interpretations, ed. P. A. Bradley, \& J. A. Guzik, ASP Conf. Ser., 135, 348

Štefl, S., Budovičová, A., Baade, D., et al. 2000, in The Be Phenomenon in Early Type Stars, ed. M. A. Smith, H. F. Henrichs, \& J. Fabregat, ASP Conf. Ser., 214, IAU Colloq., 175,240

Stefl, S., \& Rivinius, Th. 2000, in The Be Phenomenon in Early Type Stars, ed. M. A. Smith, H. F. Henrichs, \& J. Fabregat, 356

Struve, O. 1931, ApJ, 73, 94

Telting, J. H., \& Kaper, L. 1994, A\&A, 284, 515

Tubbesing, S., Rivinius, Th., Wolf, B., \& Kaufer, A. 2000, in The Be Phenomenon in Early Type Stars, ed. M. A. Smith, H. F. Henrichs, \& J. Fabregat, ASP Conf. Ser., 214, IAU Colloq., 175, 232

Underhill, A., \& Doazan, V. 1982, B Stars with and without Emission Lines, CNRS/NASA Monogr. Ser. on Nontherm. Phen. in Stell. Atmosph., NASA-SP456, 279

van Kerkwijk, M. H., Waters, L. B. F. M., \& Marlborough, J. M. 1995, A\&A, 300, 259

Waters, L. B. F. M. 1986, A\&A, 162, 121

Waters, L. B. F. M., \& Marlborough, J. M. 1994, in Pulsation, Rotation, and Mass Loss in Early-type Stars, ed. L. A. Balona, H. F. Henrichs, \& J. M. Le Contel, IAU Symp., 162 (Dordrecht: Kluwer), 399 